\title{
Medical genetic test reporting for cystic fibrosis ( $\Delta$ F508) and factor V Leiden in North American laboratories
}

Hans C. Andersson, $\mathrm{MD}^{1}$, Marie A. Krousel-Wood, MD, MSPH ${ }^{2}$, Kelly E. Jackson, MS ${ }^{1}$, Janet Rice, PhD ${ }^{3}$, and Ira M. Lubin, $P h D^{4}$

\begin{abstract}
Purpose: Physicians are ordering an increasing number of genetic tests. Results and additional information provided in the test result report are vital to the physician in making appropriate patient management decisions. Because variability in test result reports can impact patient care, we sought to determine whether variations exist in test reports for cystic fibrosis (CF) and factor $V$ Leiden (fVL) with specific comparison to professional guidelines and recommendations. Methods: A cross-sectional study design analyzing for the presence of 16 critical elements in CF reports and 12 critical elements in fVL reports solicited from United States and Canadian laboratories. Results: Of 44 laboratories performing CF testing and 72 laboratories performing fVL testing, 64\% responded. For CF reports, $21 \%$ included ethnicity, 64\% described methodology, and 61\% discussed genetic counseling. For fVL reports, $80 \%$ described methodology and 52\% discussed the need for genetic counseling in mutation-positive reports. Conclusions: Variability exists in report content among North American laboratories performing CF and fVL testing. Many reports lack information deemed critical by professional guidelines and recommendations. Genet
\end{abstract} Med 2002:4(5):324-327.

Key Words: cystic fibrosis, factor V Leiden, genetic tests, test result reporting

Molecular genetic testing has become increasingly available for diagnosis and management of various single-gene disorders such as cystic fibrosis (CF), breast cancer, and Huntington disease. ${ }^{1}$ These tests allow ascertainment of affected patients, unaffected mutation carriers, and those at increased risk for developing certain disorders. Because these mutations are often heritable, molecular genetic test results have clinical implications for other family members. ${ }^{2}$ Issues regarding informed consent, insurance implications, and pre- and post-test genetic counseling have raised concern about adequate quality assurance of molecular genetic testing. ${ }^{3,4}$

Considerable attention has been paid to the high degree of complexity involved in molecular genetic analyses. The test result report is used to convey critical information regarding the test result and other information needed by the provider in making patient management decisions. Recognizing the importance of having an appropriately informative report, sev-

From the ${ }^{1}$ Human Genetics Program, Hayward Genetics Center, Tulane University School of Medicine, New Orleans, Louisiana; ${ }^{2}$ General Preventive Medicine Residency Program, Tulane University School of Medicine and School of Public Health and Tropical Medicine, New Orleans, Louisiana; ${ }^{3}$ Department of Biostatistics, Tulane University School of Public Health and Tropical Medicine, New Orleans, Louisiana; and ${ }^{4}$ Office of Genetic Testing, DLS, PHPPO, Centers for Disease Control and Prevention, Atlanta, Georgia.

Hans C. Andersson, MD, Human Genetics Program, SL-31, Tulane University School of Medicine, 1430 Tulane Avenue New Orleans LA 70112.

Received: April 8, 2002.

Accepted: June 19, 2002.

DOI: 10.1097/01.GIM.0000029036.80969.AB eral groups have developed recommendations. The Clinical Laboratory Improvement Amendments (CLIA) is a regulatory requirement which mandates that minimum laboratory quality assurance standards be maintained. Recently, the CLIA Advisory Committee recommended the development of a genetic specialty under CLIA that, in part, will mandate specific reporting requirements. ${ }^{5}$ The National Committee for Clinical Laboratory Standards (NCCLS) has published a guideline detailing recommendations surrounding molecular diagnostic methods that addresses test result reports. ${ }^{6}$ These recommendations are listed in Table 2 of that document. The American College of Medical Genetics (ACMG) has also published recommendations and consensus documents that address reporting issues including those specific for factor V Leiden ${ }^{7}$ (fVL) and CF. ${ }^{8}$

This study was performed to assess the content variability in genetic test reports for CF $(\Delta \mathrm{F} 508)$ and fVL obtained from North American laboratories.

\section{METHODS}

Laboratories performing CF $(\Delta \mathrm{F} 508)$ and $\mathrm{fVL}$ molecular tests were identified by querying the GENETESTS ${ }^{\text {тм9 }}$ database. (Use of trade names is for identification only and does not imply endorsement by the US Department of Health and $\mathrm{Hu}-$ man Services or the US Public Health Service.) CF and fVL tests were chosen for review because they are relatively simple to perform analytically but complex to interpret. Each labora- 
tory was requested by mail (February 2000) to send the following reports with all patient identifiers removed: (1) for CF tests, a homozygous normal result, a heterozygous $\Delta \mathrm{F} 508$ result, and a homozygous $\Delta \mathrm{F} 508$ result; (2) for fVL tests, a homozygous normal result and a heterozygous affected result. Since the purpose of this study is to evaluate variability in reporting practices, rather than reporting for a specific disorder, collection of additional reports (i.e., homozygous fVL, and other CF mutations) was not considered warranted. An e-mail was sent 3 weeks after the first mailing, and a second mailing was sent to nonresponders 3 months after the first mailing. Nonresponders were telephoned 4 months after the first mailing. All laboratories were categorized as university-based, hospitalbased, or independent, depending on their affiliation with an academic institution or hospital; demographic data and response rates are presented in Table 1.

We reviewed US and Canadian test result reports for the presence of critical elements selected from the published recommendations of the Clinical Laboratory Improvement Advisory Committee (CLIAC) and NCCLS (see Table 2). Each critical element was scored once per laboratory after review of all forms. If the element was addressed in the report, the laboratory was scored as being satisfactory for that element. A report was scored positively for (1) contact information only if it included a telephone number on each form, (2) interpretation if the clinical relevance of the result was mentioned, (3) methodology if it mentioned which molecular methods were used, (4) adjusted risk if the report modified the risk for having the disorder after a negative result, and (5) genetic counseling recommendations if any mention was made on mutation-positive reports of the need to consider genetic counseling. Critical elements were categorized as administrative, patient-specific, test-specific, or post-test-specific and were scored for the percentage of all reporting laboratories including the element in their form. For the CF results, 16 elements were reviewed and for fVL results, 12 elements were reviewed (Table 2). Mutation detection rate, the mutations analyzed, revised risk assess-

Table 1

Demographics of laboratories performing molecular cystic fibrosis and factor V Leiden tests

\begin{tabular}{lcc}
\hline Test/laboratory type $(n)$ & No. (\%) responding & US/Canadian \\
\hline Cystic fibrosis & & \\
Academic (28) & $16(57)$ & $24 / 4$ \\
Hospital-based (8) & $5(63)$ & $4 / 4$ \\
Independent (8) & $7(88)$ & $7 / 0$ \\
Total $=44$ & $28(64)$ & \\
Factor V Leiden & & $39 / 2$ \\
Academic (41) & $21(51)$ & $10 / 5$ \\
Hospital-based (15) & $13(87)$ & $16 / 0$ \\
Independent (16) & $12(75)$ & \\
Total $=72$ & $46(64)$ & \\
\hline
\end{tabular}

Table 2

Summary of critical elements in cystic fibrosis and factor V Leiden test report forms

\begin{tabular}{ccc}
\hline Cystic & Factor V & \\
fibrosis & Leiden & CLIAC/NCCLS \\
$(N=28)(\%)$ & $(N=46)(\%)$ & recommended \\
\hline
\end{tabular}

Administrative elements

$\begin{array}{llcr}\begin{array}{l}\text { Medical director } \\ \quad \text { signature }\end{array} & 93 & 98 & + \\ \text { Board certification listed } & 21 & 9 & - \\ \text { Specimen collection date } & 46 & 63 & + \\ \text { Specimen received date } & 68 & 80 & + \\ \begin{array}{l}\text { Result date } \\ \begin{array}{l}\text { Contact info }+ \\ \quad \text { telephone no. }\end{array}\end{array} & 96 & 98 & + \\ \end{array}$

Patient-specific elements

Clinical indication

Ethnicity listed

Gender listed

DOB listed

$\begin{array}{ll}64 & 39 \\ 21 & \text { NA } \\ 46 & 46 \\ 79 & 80\end{array}$

Test-specific elements
Interpretation

Methodology

Mutations listed

Detection rate

Post-test-specific elements

$$
\begin{aligned}
& \text { Adjusted risk } \\
& \text { Genetic counseling }
\end{aligned}
$$

\section{(9)}

64

96

86

71

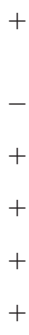

\begin{tabular}{l}
+ \\
+ \\
- \\
+ \\
+ \\
+ \\
+ \\
+ \\
+ \\
+ \\
\hline
\end{tabular}

Percentage of responding laboratories including critical elements on test report forms for cystic fibrosis and factor $\mathrm{V}$ Leiden testing and the identification of items recommended by CLIAC or NCCLS (+). NA, not applicable.

ments based on number of mutations tested, and ethnicity were scored for CF only because fVL testing involves a single panethnic mutation. We used the $\chi^{2}$ test of independence to explore the associations among the variables. When the expected frequencies were small, we used the Fisher exact test.

\section{RESULTS}

For CF, 28/44 laboratories responded (64\%) and for fVL, $46 / 72$ laboratories responded (64\%; Table 1). No differences in response rates were found between the two types of tests $(P=$ 0.98). No significant differences in response rate were found among the types of laboratories for CF $(P=0.29)$, but significantly fewer academic laboratories responded than hospitalbased or independent laboratories for fVL $(P=0.03)$.

Table 2 shows the results of analyzing the test report forms for the $16 \mathrm{CF}$ elements and the $12 \mathrm{fVL}$ elements that were evaluated. Almost all laboratories included a medical director's signature (93\% [CF] and 98\% [fVL]), but very few listed board 
certification status of the medical director $(21 \%[\mathrm{CF}]$ and $9 \%$ [fVL]). Contact information was offered by $86 \%$ and $87 \%$ of laboratories for $\mathrm{CF}$ and $\mathrm{fVL}$, respectively. Patient ethnicity was given by only $21 \%$ of CF laboratories. Test methodology was included in $64 \%$ of CF laboratories and $80 \%$ of fVL laboratories. Genetic counseling was mentioned in mutation-positive reports by $61 \%$ and $54 \%$ of CF- and fVL-performing laboratories, respectively. CF reports were more likely to include clinical indication than were fVL reports $(P<0.01)$, but fVL reports were more likely to include methodology $(P<0.05)$. No other significant differences in the report features were observed between the two disorders.

\section{DISCUSSION}

As with other clinical tests, appropriate clinical interpretation and use of the genetic test result often requires additional information provided by the laboratory. This is particularly pertinent because genetic testing not only includes tests referred for diagnostic purposes but also includes tests to determine disease susceptibility and carrier status. Furthermore, implications for other family members is an inherent part of results established for most genetic tests and will depend on possible modes of inheritance, penetrance, and expressivity of the mutations found. Using CF and fVL as model conditions, we sought to assess the presence of certain critical elements that impact interpretation of the results and impact patient management decisions. Variability has been previously noted in CF testing. ${ }^{10}$ This together with its potential to be used for population-based screening has led the $\mathrm{ACMG}^{8}$ to develop recommendations that address various aspects of CF testing including the reporting of results. McGovern et al. ${ }^{3}$ reported that a significant number of laboratories do not request a family history on their requisition and did not provide a summary of the method used to perform the analysis. Giardello et al. ${ }^{4}$ additionally found that $32 \%$ of physicians in their study misinterpreted the test result. Given these findings and the belief that these elements are necessary for appropriate interpretation of the test result, several groups have proposed the inclusion of critical elements in genetic test result reports but no systematic review of test reporting practices has been performed. ${ }^{5,6}$

The present study may be limited by our ability to identify all laboratories offering CF and fVL testing and the response rate. Nonetheless, the responses allowed us to perform a qualitative assessment of report variability. In addition, report forms for these two disorders may not fully represent the spectrum of interpretative reports from a laboratory.

We found a broad range of reporting content, with considerable variance from the recommended guidelines noted in the critical elements included in the report. A high degree of similarity existed between the percentage of report forms for $\mathrm{CF}$ and fVL test responding adequately to any given critical element. This similarity suggests that inclusion of critical elements on the report form is not test-specific but may be related to general laboratory report practices. For example, almost all laboratories performing either test included a medical direc- tor's signature but few included information regarding genetic board certification of the medical director. The presence of a board-certified geneticist and laboratory accreditations were two of the factors previously correlated with optimal practices, ${ }^{3}$ although they are not specifically recommended for inclusion on report forms by CLIAC and NCCLS.

In $36 \%$ and $20 \%$ of CF and fVL reports, respectively, no mention was made of the methodology used in the test. Laboratory methodology affects the clinical interpretation of the result. A negative screen for some mutations does not rule out the possibility of carrying a disease-causing mutation, and omission of the methodology may lead to a misunderstanding that potentially adversely affects clinical management. Specifically in CF, a report failing to list methodology may be misinterpreted as analyzing sweat electrolytes rather than mutation analysis. As methodologies change, mutation ascertainment may change and the post-test risk of carrying a disease-specific mutation must be adjusted.

The clinical indication for performing the test was mentioned by $64 \%$ and $39 \%$ of CF- and fVL-performing laboratories, respectively. The ACMG recommends a standard mutation panel for CF screening, including R117H. ${ }^{8}$ The presence of $\mathrm{R} 117 \mathrm{H}$ can be associated with congenital bilateral absence of the vas deferens (CBAVD) when present in cis with a $7 \mathrm{~T}$ splice variant and a second CF mutation or trans with a $5 \mathrm{~T}$ splice variant. ${ }^{11}$ Many of these patients may not have elevated sweat chloride concentrations or lung disease typical of CF. Alternatively, when this mutation is present in cis with a $5 \mathrm{~T}$ splice site variant and a CF mutation on the other allele, a pancreatic sufficient form of CF can result. Therefore, knowing the clinical reason and indications for testing, whether for CF diagnostic testing, carrier testing, or addressing a clinical finding of CBAVD, is needed in order to provide an appropriate interpretation and recommendations for follow-up.

Only $21 \%$ of CF-performing laboratories mentioned the ethnicity of the patient, a factor that may significantly affect the interpretation of the result since the $\Delta$ F508 mutation is most frequent among populations derived from northern Europeans. Ethnicity is necessary for calculating a post-test adjusted risk of carrying a mutation when a mutation is not found on one or both alleles. Therefore, without identifying ethnicity, a physician ordering a CF mutation analysis for carrier status who receives a "negative" result might incorrectly counsel the patient that he or she is not a carrier or over- or underestimate the residual risk from the ethnicity-specific detection rates. The carrier frequency for the $\Delta \mathrm{F} 508$ allele varies among ethnic groups (from approximately $70 \%$ of contributing alleles in Caucasians, $46 \%$ in one Hispanic population, $48 \%$ in African Americans, and 30\% in Ashkenazi Jews) and, therefore, knowledge of ethnicity is critically important in calculating residual risk. ${ }^{8}$ Because of this variation in the prevalence of the $\Delta \mathrm{F} 508$ allele, both the detection rate and estimated carrier risk after a negative test result will vary. For example, in European Caucasians, the overall detection rate using the ACMG recommended test panel is estimated at $80 \%$ with a 1 in 140 estimated carrier risk after a negative test result (to include all contribut- 
ing alleles). In the Hispanic population studied, the detection rate was reported at 57\% with a 1 in 105 estimated carrier risk after a negative test result. ${ }^{12}$ Alternatively, although the prevalence of $\Delta$ F508 is relatively low in the Ashkenazi Jewish population, other prevalent alleles allow for a $97 \%$ detection rate. Although it may be debated whether reporting the ethnicity on the result report is necessary, an indication regarding how detection rate and residual risk calculations were performed should be helpful in assuring the referring clinician that the numbers reported are appropriate for the patient referred.

Only $61 \%$ and $54 \%$ of CF and fVL mutation-positive reports, respectively, mentioned the consideration of genetic counseling for other family members. Ideally, genetic counseling should have been provided prior to testing but failure to provide or recommend genetic counseling post-test may result in a lack of understanding and action on the part of the patient regarding his or her carrier status and risk for other family members. Inclusion of a recommendation for genetic counseling in the laboratory report emphasizes this intervention in patient care.

The widespread use of genetic tests by physicians without specialized genetic training has raised concern about the appropriateness of test ordering and the adequacy of test reporting. ${ }^{2,4}$ Our study results suggest that information which may affect the physician's management of the patient (e.g., test methodology, post-test adjusted risk for being a mutation carrier, consideration of genetic counseling) is not uniformly included in CF and fVL test reports. These findings confirm that some medical genetic test reports are currently not providing a complete set of informative elements for ordering physicians. We recognize that a laboratory's capacity to include information in its report depends on what is asked for (or required) in the requisition and the willingness of physicians to provide the requested information.

In summary, we identified variability of reporting content in the current medical genetic laboratory reports for CF and fVL testing. We suggest that these findings prompt a review by genetic laboratories of their current test reports to consider inclusion of the critical elements, which are necessary for all physicians to appropriately interpret and use the report results. Future areas of investigation will include a study to identify barriers to effective understanding of genetic test result reports by general and specialty physicians. These efforts will ultimately lead to recommendations for broadly applicable laboratory reporting practices that permit the physician to make appropriate clinical interpretations with a high degree of confidence.

\section{Acknowledgments}

This study was supported by a cooperative agreement with the Association of Teachers of Preventive Medicine, the Centers for Disease Control and Prevention, and the Agency for Toxic Substances and Disease Registry.

\section{References}

1. Holtzman NA, Murphy PD, Watson MS, Barr PA. Predictive genetic testing: from basic research to clinical practice. Science 1997;278:602-605.

2. Grody WW, Pyeritz RE. Report card on molecular genetic testing: room for improvement? JAMA 1999;281:845-847.

3. McGovern MM, Benach MO, Wallenstein S, Desnick RJ, Keenlyside R. Quality assurance in molecular genetics testing laboratories. JAMA 1999;281:835-840.

4. Giardiello FM, Brensinger JD, Petersen GM, Luce MC, Hylind LM, Bacon JA, Booker SV, Parker RD, Hamilton SR.. The use and interpretation of commercial APC gene testing for familial adenomatous polyposis. N Engl J Med 1997;336:823827.

5. Genetic testing under the Clinical Laboratory Improvement Amendments. Fed Regist 2000;65:87.

6. National Committee for Clinical Laboratory Standards. Molecular Diagnostic Methods for Genetic Diseases; Approved Guidelines. National Committee for Clinical Laboratory Standards 2000; CCLS document MM1-A, Vol 17.

7. Grody WW, Griffin JH, Taylor AK, Korf BR, Heit JA. American College of Medical Genetics Consensus statement on factor V Leiden mutation testing. Genet Med 2001;3:139-148.

8. Grody WW, Cutting GR, Klinger KW, Richards CS, Watson MS, Desnick RJ. Laboratory standards and guidelines for population-based cystic fibrosis carrier screening. Genet Med 2001;3:149-154.

9. GeneClinics. Medical genetics information resource: University of Washington and Children's Health System, 2001. http://www.genetests.org.

10. Grody WW, Desnick RJ, Carpenter NJ, Noll WW. Diversity of cystic fibrosis mutation screening practices. Am J Hum Genet 1998;62:1252-1254.

11. Kiesewetter S, Macek M Jr, Davis C, Curristin SM, Chu CS, Graham C, Shrimpton AE, Cashman SM, Tsui LC, Mickle J. A mutation in CFTR produces different phenotypes depending on chromosomal background. Nat Genet 1993;5:274-278.

12. Cutting GR. Genetic epidemiology and genotype/phenotype correlations. NIH Consensus Development Conference on Genetic Testing for Cystic Fibrosis, Bethesda, MD, April 14-16, 1997. 